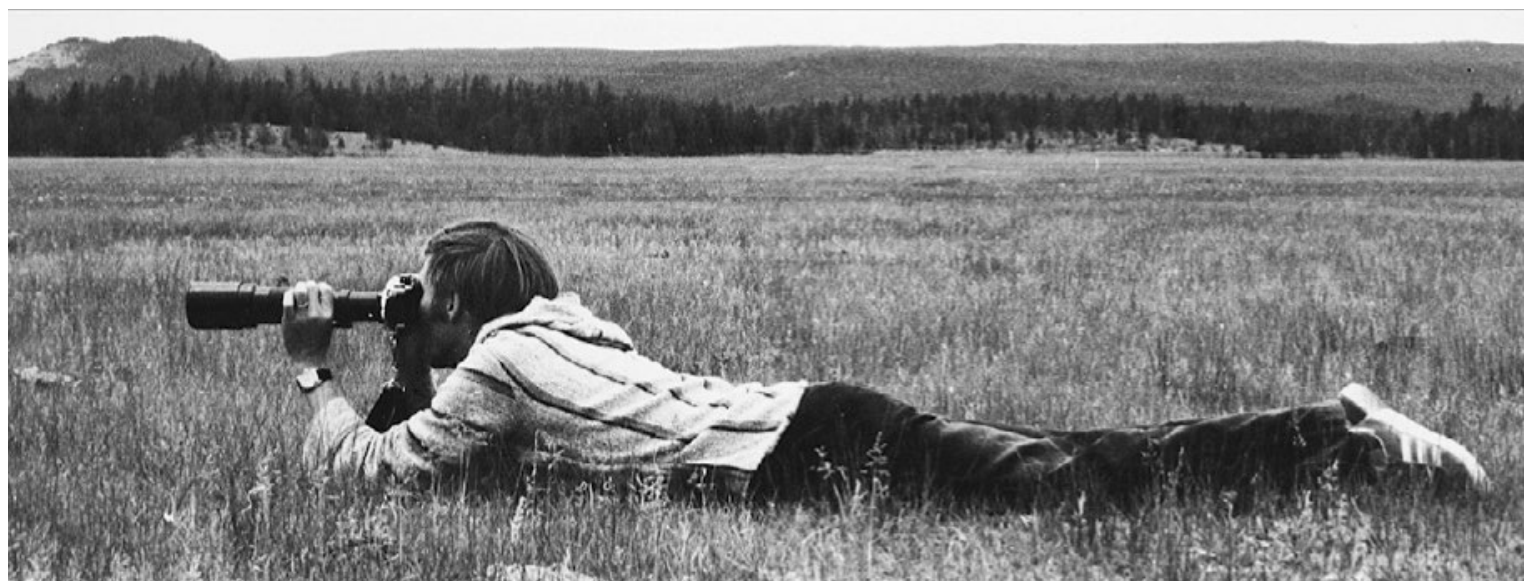

Auf der Büffelpirsch in den USA.

\title{
Eminenz, Evidenz, Exzellenz
}

\section{Matthias Scholer}

Wissenschaftsjournalist

Ein Mann liegt bäuchlings im Gras, seine Kamera mit markantem Teleobjektiv auf ein nicht sichtbares Objekt gerichtet. Dies ist eines der auffallend wenigen Bilder, die mir die Suchmaschine nach der Eingabe des Namens Hans Rudolf Koelz liefert. Wie dieses Bild wohl zur Person passt, die ich heute zum Interview treffen werde? Zumindest ist das Motiv unerwartet unüblich für einen Professor der Gastroenterologie und langjährigen Vizepräsidenten des SIWF.

Herr Koelz, wann ist das obenstehende Foto entstanden?

Hans Rudolf Koelz: Das Foto hat meine Frau vor rund 40 Jahren im Yellowstone Nationalpark aufgenommen, als ich mich an einen Bison heranpirschte. Es ist für meine Person charakteristisch.

\section{Inwiefern?}

Seit meiner Schulzeit interessiere ich mich sehr für Naturwissenschaften, aber auch für Musik. Zuerst liebäugelte ich mit einem Studium der Zoologie oder der Veterinärmedizin. Mir wurde jedoch rasch klar, dass ich danach kaum reelle Chancen haben würde, Zoodirektor zu werden, sondern wohl irgendwo als Lehrer enden würde. Also ging ich zum Berufsberater. Der konnte mir jedoch nicht weiterhelfen. Er meinte bloss, dass sämtliche Studien für mich in Frage kämen. So entschied ich mich schliesslich für die Humanmedizin, da mir diese Ausbildung die meisten Optionen offenliess.

Sie haben bereits vor dem Abschluss ihres Studiums ein Diplom in Tropenmedizin gemacht.

Also schlummerte doch ein Hauch von Daktari und Albert Schweitzer in Ihnen?

Tatsächlich träumte ich während des Studiums von einem Einsatz in den Tropen. Diese Leidenschaft teilte ich mit einem engen Freund, den ich schon von der Schulzeit her kannte. Wir beide langweilten uns etwas während des Studiums. Und so absolvierten wir parallel zum regulären Unterricht den Tropenkurs am Schweizer Tropeninstitut. Dabei kam ich dank interessanten Themen wie Gifttierkunde, Veterinärmedizin und Biologie voll auf meine Rechnung. 


\section{Trotzdem waren Sie danach nicht als Tropenarzt tätig?}

Ja, es kommt eben doch oft anders, als man denkt. Zwei Wochen nach dem Staatsexamen reiste ich als Expeditionsarzt einer alpinistischen Expedition auf dem Landweg von Zürich nach Kabul und dann in den Hindukusch. Ich kehrte danach in die Schweiz zurück.

\section{Evidenz statt Eminenz}

Während Hans Rudolf Koelz seine Jugenderinnerungen schildert, erkennt man in seinen Augen das Feuer, welches den jungen Mediziner angetrieben hat, möglichst viel Wissen oder vielmehr Verstehen in sich aufzusaugen. Fallen bestimmte Schlüsselwörter, driftet unser Gespräch gerne in eine neue, nicht minder spannende Richtung. Ein Begriff auf den wir dabei immer wieder stossen ist die «Evidenz-basierte Medizin».

\section{Nach zwei Jahren als Assistenzarzt wechselten Sie in den Forschungsbereich. Wie kam es dazu?}

Während meiner Zeit an der Medizinischen Klinik des Stadtspitals Triemli lernte ich Professor André Blum kennen. Er nahm mich im Rahmen eines Nationalfondsprojekts im Bereich der Gastroenterologie unter seine Fittiche. Blum war für mich persönlich und meinen beruflichen Werdegang prägend. Bei ihm lernte

«So lernte ich schon früh, dass jede Behauptung in einer wissenschaftlichen Arbeit mit Fakten, Zahlen und Beweisen unterlegt sein muss.»

ich nicht nur, wissenschaftlich zu denken. Er vermittelte mir auch, weshalb die dazumal populäre «eminence-based medicine» ausgedient hatte und durch «evidence-based medicine» ersetzt werden musste. So lernte ich schon früh, dass jede Behauptung in einer wissenschaftlichen Arbeit mit Fakten, Zahlen und Beweisen unterlegt sein muss. Zwar braucht es dafür nicht immer randomisierte Doppelblindstudien, aber Schlüsse aus persönlichen Erfahrungen mit $n=1$ zu ziehen, hatten für mich damit definitiv ausgedient.

\section{Viele Behandlungsmethoden kommen trotz mangelnder Evidenz zum Einsatz ...}

Es gibt diagnostische und therapeutische Methoden, bei denen es kaum möglich ist, alles zu testen und zu belegen. Trotzdem machen sie Sinn. Aber es existieren tatsächlich Gebiete, in denen die Evidenz, die nach allen Regeln der Kunst erforscht wurde, klar zeigt, dass etwas nicht funktioniert. Dass solche Behandlungsmethoden trotzdem im klinischen Alltag angewendet und von den Kassen sogar vergütet werden, ist störend.

\section{Können Sie uns ein Beispiel dafür geben?}

Die Homöopathie ist ein gutes Beispiel. Wenn man behauptet, ein Arzneimittel habe eine stärkere Wirksamkeit als ein Placebo, obwohl die Moleküle, welche bei der Produktion ins Wasser gegeben wurden, nicht mehr vorhanden sind, dann will ich dafür solide Beweise. Irgendjemand hat mal gesagt: «Für ausserordentliche Behauptungen, braucht es ausserordentlich gute Evidenz.» Dem kann ich nur zustimmen. Ohne entsprechende Evidenz lasse ich mir mein Weltbild nicht so leicht umkrempeln.

\section{Weiter- und Fortbildung}

Die Mitarbeit an einem Nationalfondsprojekt weckte den Wissenschaftler in Hans Rudolf Koelz. Nach seiner Zeit in Zürich folgten einige Jahre in den USA, während derer Koelz im Bereich der Gastroenterologie forschte. Oder wie er es augenzwinkernd ausdrückt: «Ich pipettierte etwa sechs Jahre lang.» Nach seiner Rückkehr in die Schweiz setzte Hans Rudolf Koelz seine Karriere als praktizierender Gastroenterologe fort. Parallel zur klinischen Tätigkeit engagierte sich Koelz vermehrt auch im Bereich der Weiter- und Fortbildung. Dies insbesondere beim Schweizerischen Institut für ärztliche Weiter- und Fortbildung (SIWF) bzw. dessen Vorgängerorganisation. Koelz amtete die letzten acht Jahre seiner 24-jährigen Mitarbeit in diesen Gremien als Vizepräsident des SIWF.

\section{Was gehörte zu Ihren Aufgaben als Vizepräsident des SIWF?}

Ich kümmerte mich hauptsächlich um die Weiterbildungsprogramme. So musste ich Anträge auf neue Weiterbildungsgänge verschiedenster Fachrichtungen auf ihre Sinnhaftigkeit hin überprüfen. Nach meiner Begutachtung folgte die Einschätzung durch die Geschäftsleitung. Auch wenn während dieses Prozesses der Diskurs mit den zuständigen Fachgesellschaften gesucht wird, fällt schliesslich der Vorstand, seltener das Plenum des SIW, demokratisch den Entscheid, ob ein Antrag angenommen wird oder nicht. Dank der weitgehenden Eigenständigkeit des SIWF sind die Experten in ihrer Entscheidungsfindung soweit machbar unabhängig von gesundheits-, standespolitischen und pekuniären Gegebenheiten. Damit lassen sich Änderungen im Gefüge der Weiter- und Fortbildung effizient umsetzen - ein Umstand, für den wir von unseren europäischen Kollegen in analogen Gremien häufig beneidet werden.

Was steht der Entwicklung der Weiterbildung im Weg? Das Arbeitsgesetz schränkt die Weiterbildung stark ein. Assistenzärztinnen und -ärzte können kaum mehr zu- 


$\begin{aligned} & \text { Stark verkürzter Lebenslauf } \\
& \text { von H. R. Koelz }\end{aligned}$
\begin{tabular}{ll}
1972 & Abschluss Medizinstudium Basel \\
\hline $1973-1986$ & $\begin{array}{l}\text { Verschiedene Stationen im Bereich der Inneren } \\
\text { Medizin und Forschung auf dem Gebiet der } \\
\text { Gastroenterologie (Schweiz und USA). }\end{array}$ \\
\hline 1986 & $\begin{array}{l}\text { Habilitation Universität Bern, Facharzt Innere } \\
\text { Medizin und Gastroenterologie. }\end{array}$ \\
\hline $1986-2009$ & $\begin{array}{l}\text { Leiter der Abteilung Gastroenterologie, Stadt- } \\
\text { spital Triemli und diverse Funktionen in Verbän- } \\
\text { den und Kommissionen. }\end{array}$ \\
\hline $1992-2000$ & $\begin{array}{l}\text { Mitglied des Plenums der Kommission für Wei- } \\
\text { ter- und Fortbildung (KWFB) der FMH }\end{array}$ \\
\hline $2000-2008$ & Mitglied des Ausschusses der KWFB der FMH \\
\hline $2008-2016$ & $\begin{array}{l}\text { Vizepräsident des Schweizerischen Instituts für } \\
\text { Weiter- und Fortbildung (SIWF). }\end{array}$ \\
\hline
\end{tabular}

In seiner Karriere publizierte Hans Rudolf Koelz rund 180 wissenschaftliche Arbeiten.

sätzliche Vorlesungen oder Kongresse besuchen, ohne die maximal zulässige Wochenarbeitszeit von 50 Stunden zu überschreiten. Das muss unbedingt geändert werden. Wir haben vorhin von Evidenz gesprochen. Für die Behauptung, jemand könne nach 50 Stunden nicht mehr adäquat arbeiten, liegt diese bei Null.

\section{"All diese Faktoren zusammen machen die Aus- und Weiterbildung in einer Kosten-Nutzen- Bilanz sehr teuer.»}

Verschärfend kommt hinzu, dass immer mehr Frauen Medizin studieren. Viele von ihnen sind nach dem Erreichen des Facharzttitels jedoch nicht mehr ärztlich tätig, oder arbeiten wie die Mehrheit der männlichen Kollegen nur noch Teilzeit. All diese Faktoren zusammen machen die Aus- und Weiterbildung in einer Kosten-Nutzen-Bilanz sehr teuer.

\section{Sie haben letztes Jahr ihr Amt an Giatgen Spinas übergeben. Ist damit das Kapitel SIWF für Sie abgeschlossen?}

Ich bin sehr froh, dass das SIWF einen so kompetenten Nachfolger gefunden hat. Was mich noch etwas beun-

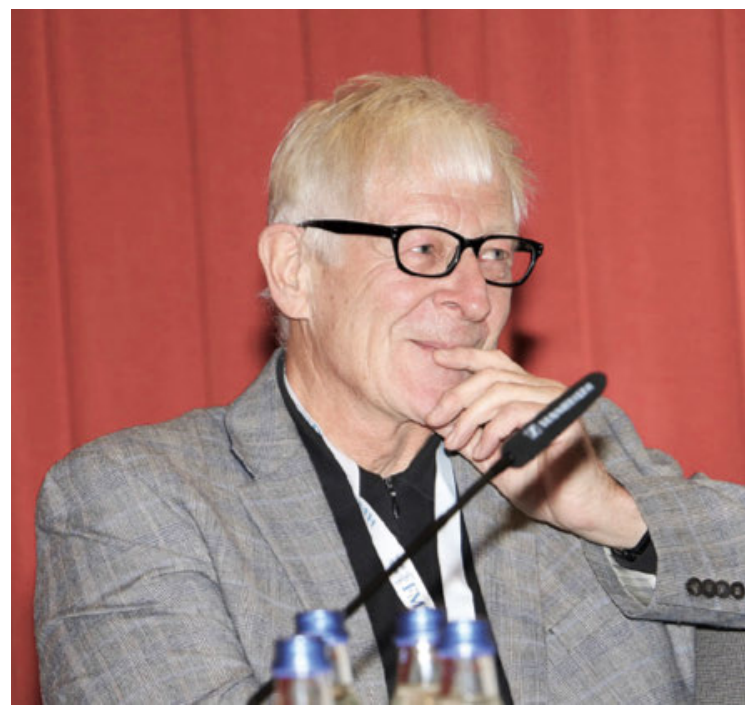

Hans Rudolf Koelz kümmerte sich jahrelang beim SIWF um die Weiterbildung.

ruhigt, ist die Tatsache, dass noch keine Nachfolge für den Präsidenten Werner Bauer am Horizont zu sehen ist. Dabei wäre es doch so wichtig, dass diese Person von Bauers unglaublich breitem Fachwissen profitieren könnte.

Zum Schluss unseres Gesprächs frage ich Hans Rudolf Koelz noch, ob es ihm nicht schwerfällt, nach einem so abwechslungsreichen Berufsleben kürzer zu treten. Doch als er mir dann von seinem Garten, seinen «kühnen» Wanderungen und der Zuneigung zur Musik erzählt, zerstreuen sich meine Bedenken, dass sich Koelz mit dem Ruhestand schwertut.

Übrigens habe er sich, so erzählt er mir, auch gerade ein Gerät zusammengebaut, mit dem er den Ruf der Fledermäuse hörbar machen könne. So liegt Hans Rudolf Koelz nun nicht mehr mit dem Teleobjektiv auf der Lauer, dafür schwenkt er ein Richtmikrofon in den Nachthimmel.

\section{Bildnachweis}

Schwarzweissfoto: Hans Rudolf Koelz

Porträt: Bruno Kesseli 